\title{
A Low Cost Tele-Rehabilitation Device for Training of Wrist and Finger Functions After Stroke
}

\author{
Patrick Weiss \\ Institute of Computer \\ Engineering \\ Graduate School for \\ Computing in Medicine and \\ Life Sciences \\ University of Lübeck \\ Ratzeburger Allee 160, \\ 23562 Lübeck, Germany \\ weiss@iti.uni-luebeck.de \\ Marcus Heldmann \\ University Medical Center \\ Schleswig-Holstein \\ University of Lübeck \\ Ratzeburger Allee 160, \\ 23562 Lübeck, Germany \\ marcus.heldmann@ \\ neuro.uni-luebeck.de
}

\author{
Alexander Gabrecht \\ Institute of Computer \\ Engineering \\ University of Lübeck \\ Ratzeburger Allee 160, \\ 23562 Lübeck, Germany \\ gabrecht@iti.uni- \\ luebeck.de
}

\author{
Achim Schweikard \\ Institute for Robotics and \\ Cognitive Systems \\ University of Lübeck \\ Ratzeburger Allee 160, \\ 23562 Lübeck, Germany \\ schweikard@rob.uni- \\ luebeck.de
}

\author{
Thomas M. Münte \\ University Medical Center \\ Schleswig-Holstein \\ University of Lübeck \\ Ratzeburger Allee 160, \\ 23562 Lübeck, Germany \\ thomas.muente@neuro.uni- \\ luebeck.de
}

\author{
Erik Maehle \\ Institute of Computer \\ Engineering \\ University of Lübeck \\ Ratzeburger Allee 160, \\ 23562 Lübeck, Germany \\ maehle@iti.uni- \\ luebeck.de
}

\begin{abstract}
There is a need for robotic rehabilitation devices that improve the outcome while reducing the cost of therapy. This paper presents a device for training of supination / pronation, dorsal wrist extension, and finger manipulation after stroke. The system exhibits modularity in terms of the communication architecture and different optional components. User interfaces (UI) can be implemented on different kinds of devices including a Rasperry Pi single-board computer on which a Qt-based graphical UI was run in this instance. Tele-rehabilitation functionality is included using SSL-encrypted RESTful web services on a three-tier architecture. Expensive sensors were omitted in order to have a costeffective system which is a requirement for home-based rehabilitation. The current-based torque sensing is evaluated by comparing current measurements to force-torque sensor values. After canceling out the static friction, the low error justified the omission of an additional sensor.
\end{abstract}

\section{Categories and Subject Descriptors}

Applied computing [Life and medical sciences]: Consumer health

\section{General Terms}

Design, Measurement, Performance

Permission to make digital or hard copies of all or part of this work for personal or classroom use is granted without fee provided that copies are not made or distributed for profit or commercial advantage and that copies bear this notice and the full citation on the first page. To copy otherwise, to republish, to post on servers or to redistribute to lists, requires prior specific permission and/or a fee. REHAB 2014, May 20-23, Oldenburg, Germany Copyright (C) 2014 ICST 978-1-63190-011-2

DOI 10.4108/icst.pervasivehealth.2014.255331

\section{Keywords}

Robotic rehabilitation, tele-rehabilitation, stroke, wrist and finger functions, home health care

\section{INTRODUCTION}

In high-income countries, stroke is the third most common cause of death and the major cause of acquired adult disability [14]. Besides personal consequences, this means a high economical impact. The average lifetime cost for stroke rehabilitation per case in Germany is 43,129 EUR and projected 3.4 million new cases of ischemic stroke from 2006 to 2025 lead to estimated costs of 108.6 billion EUR [5].

Often, the upper limb is affected leading to significant problems in fine and gross motor skills. After three months, only $20 \%$ to $56 \%$ of all stroke survivors regain useful upper limb function [10]. In robotic therapy, training of the distal parts of the upper-limb of the wrist appears to be a crucial factor in this recovery. A wrist extension for robotic upper-limb therapy device further increased the rehabilitation outcome in comparison to training of the shoulder and elbow alone [6]. Reaching and wrist supination/pronation training is particularly important since it promotes progress towards more functional whole upper extremity movements [11].

Several robotic systems for upper limb rehabilitation have been presented [8]. While some studies show promising results in outcome [13], they are not consistently in favor of robotics [7]. This underlines the necessity for a better understanding of motor learning which can be enhanced by means of robotic rehabilitation [12].

Seen from another perspective, these systems do not have to compete against traditional therapy, but may instead be 
complimentary, e.g. by deploying rehabilitation devices in the home environment. Home rehabilitation potentially reduces costs by increasing the amount of independent training time and relieving the load of therapists. Telerehabilitation gives them a means of control and surveillance over the training and the possibility of intervention if necessary.

However, most of the proposed devices are not suitable for home rehabilitation. Many systems are either too expensive, e.g. due to the use of force-torque sensors [9], do not offer modularity and virtual rehabilitation [4], or are fitted to other systems [1]. Nearly $75 \%$ of the devices observed in a comprehensive review have not even undergone any sort of testing due to high complexity and poor usability [2].

Based on the aforementioned points, we propose a device called $m \cdot R e S^{R 2}$ (second prototype of a modular Rehabilitation System for training of Rotational movements) for training of wrist (supination / pronation / dorsal extension) and finger functions focusing on its application in the home environment. The system is required to be cost-effective, and to provide an intuitive user interface. Essential to its function are an actuator and sensors for virtual rehabilitation and the study of rehabilitation paradigms, particularly visual feedback distortion [3], and the ability to give remote access to session data for tele-rehabilitation.

\section{CONCEPT AND IMPLEMENTATION 2.1 Concept and Design}

There are two basic approaches of training devices: Endeffectors and exoskeletons. Rotational movements involve a high number of degrees of freedom of the hand, which exoskeletons have to provide to allow fully unconstrained movements. Following a cost-effective approach, we chose an end-effector design which allows tasks involving many DOF without the need for a high number of mechanical DOF.

Depending on the orientation of the device and the handle used, different movements can be trained. Supination and pronation exercises are performed in the horizontal position with the arm aligned to the motor axis. Dorsal wrist extension and fine finger exercises require the device to be orientated vertically (see Fig. 1). To this effect the motor axis lies perpendicular to the arm such that the method of interaction changes and additional muscles are activated. This way, only one actuator is necessary reducing complexity and cost, while still allowing for different training methods. The whole device can be adapted in height and orientation using an adjustable table. A chuck attached to the motor can fasten different handles giving more flexibility in training. Supination/pronation requires an additional handle other than dorsal wrist extension and by varying its size, the difficulty for fine manipulation training can be tuned.

The end-effector's main component is a motor that supports or impedes the patient in performing rotational movements. The motor is placed within a casing that also houses the power supply with an emergency switch, a microcontroller board, electronics, and a control panel. Optionally, a singleboard computer (Rasperry Pi) may be included.

Different training modes are provided. In passive mode, the patient is mobilized either by continuously following

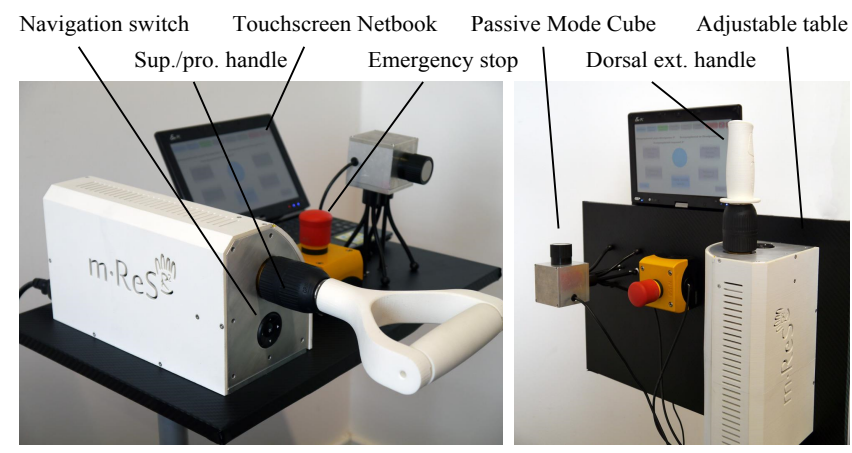

Figure 1: Horizontal (1.) and vertical (r.) orientation of the training device

a sine trajectory with adjustable amplitude and frequency or by using the passive mode cube. This optional module allows hemiparetic patients to control the motor by turning a knob with their healthy hand [15]. Depending on the progress of the patient, the support can be reduced or counter-torques can be applied. The range of motion and torque measurements are compared over sessions to give feedback on the progress. Simple rehabilitation games have been implemented to make training more diverse. Visual feedback distortion can be applied which is subject to studies of the cooperating neurologists.

\subsection{Actuation, Sensing, and Electronics}

A brushed motor and a gear with a reduction ratio of $7: 1$ were used achieving a maximum total torque of $1.7 \mathrm{Nm}$. The combination of a strong motor with a low reduction gear ratio results in low backdrivability while achieving a decent maximum torque. It may not be sufficient to work against strong spasms but safety concerns and backdrivability outweighed this possibility. An encoder with 1024 impulses per revolution delivers the relative angle in incremental steps of $0.05^{\circ}$. A motor driver amplifies the pulse-width modulation (PWM) signals from the microcontroller.

Torque measurements are an important factor in assessing the patient's capabilities. Force-torque sensors were not an option, since they cost many times more than the presented system. Therefore, the torque measurement utilizes the linear relationship to the armature current. A Hall effect based current sensor IC converts the current to voltage measured by the microcontroller. The maximum torque can then be restricted by comparing the measured current to a set point and adjusting the PWM signal with an integrative controller. A serial connection between the microcontroller and a user interface device is established either tethered, using a USB to UART bridge, or wireless based on a Bluetooth module with Serial Port Profile (SPP).

\subsection{Software and Communication}

The software is designed in a modular way. It consists of a three-tier architecture to provide tele or home rehabilitation capabilities. The client, located at the patient's side, gathers data for the evaluation of the rehabilitation progress. For comparison of the training outcome, the patient's maximum range of motion and exerted torque are logged for every training or game session. Furthermore, the trajectory of 


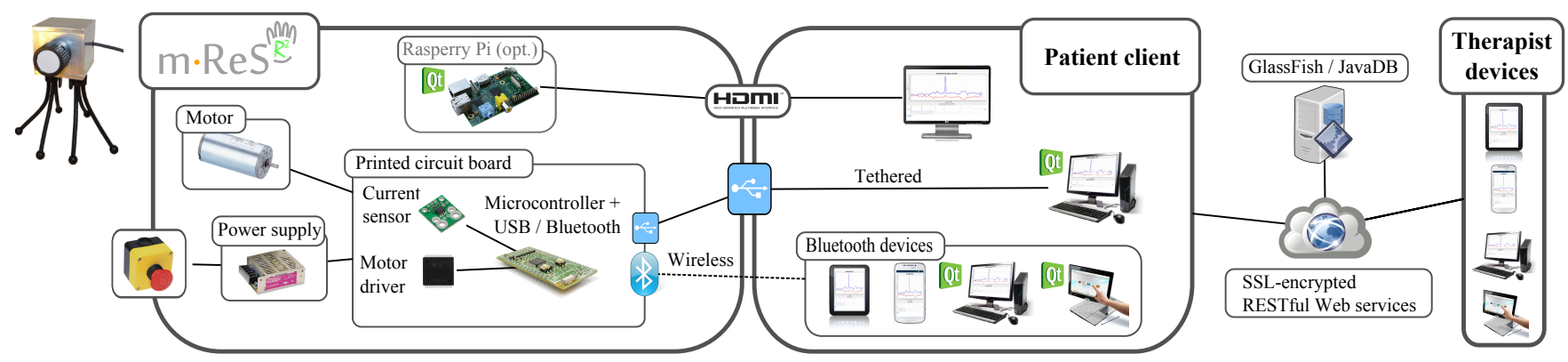

Figure 2: Schematic of the system and possible interaction platforms

each session is stored to provide data which can be useful to examine intrasession effects like fatigue.

To maximize the flexibility in client hardware, our prototype client software is a cross-platform implementation based on Qt4 which runs on standard PCs (Windows, Mac OS X, Linux) as well as on single-board computers like the Raspberry $\mathrm{Pi}$. We ported and natively compiled our software on a Rasperry Pi Model-B running Raspbian Wheezy to provide an all-in-one client. The software includes a GUI for progress measurements, motivating rehabilitation games and progress visualization. The interface can alternatively be controlled by the integrated navigational switch panel, touchscreen or standard PC input devices (keyboard/mouse).

The communication architecture allows for a variety of platforms to access the functionality and visualize sensor data. The client connects to the microcontroller over a serial UART connection to gather data or set parameters like resistance or target position. The data of each training session is transmitted to the clinic side server or cached locally while a server connection is not available.

The server software is hosted on a Glasfish application server and implements a SSL-encrypted RESTful web service which receives data from training sessions and provides configuration values to the client software like targets. It consists of a web front-end based on PrimeFaces and connects to a JavaDB database. The RESTful approach combined with the PrimeFaces UI ensures compatibility with various client and web-browsers. The interface features administrative, therapists', and patient views with restricted rights.

\section{PERFORMANCE EVALUATION}

The first experiment evaluates the accuracy of the torque estimation from the current measurement. We use a forcetorque sensor that is rigidly connected to the device. A current profile is applied on the end-effector and the estimated torque is compared to the measured one. The profile includes a step at the beginning and a drop at the end of the sampling period. These two points are used for synchronization and scaling of the two sensors that return samples with constant but different frequencies. The values after the initial step and before the increase of current define the baseline on which the force-torque sensor is calibrated. The results are plotted in Fig. 3.

The dotted line represents the initial setpoint current which

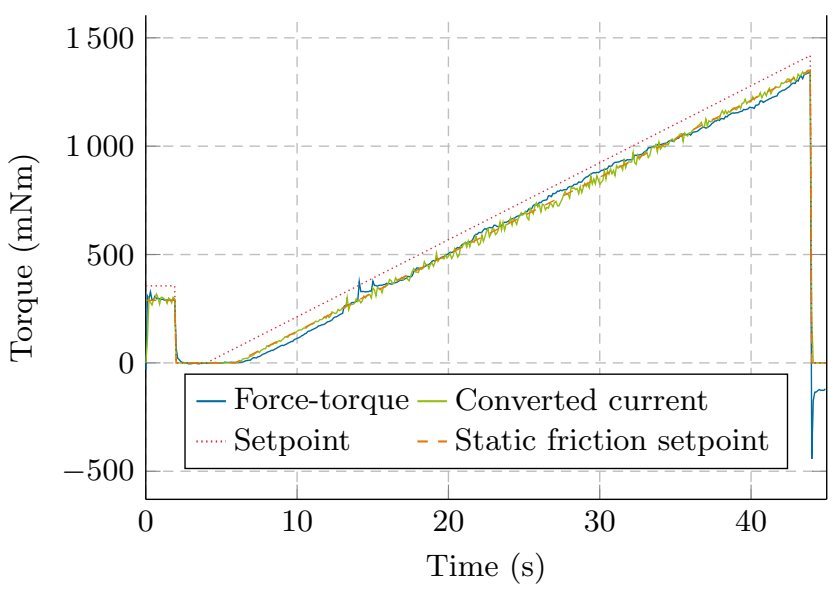

Figure 3: Comparison of force-torque to current measurements including the setpoint trajectory with and without regarding the static friction. All currents are converted with the torque constant determined by the force-torque test.

is converted using the linear relation between torque and current. At the beginning of the slope, the increase of current does not result in higher torque. We blame unwanted influences of the gear, mostly by static friction, for the reduced transmitted torque. Therefore, we determine the static friction together with the torque constant by fitting a linear model with $T(i)=k_{m} i+b$, where $T$ is torque, $i$ is current and $k_{m}$ is the torque constant, to the torque measurement. Iteratively, we increase the static friction and repeat the fitting without the values below the threshold until b is approximately zero. Thus, the static friction was determined to have a threshold current of $188 \mathrm{~mA}$. The slope represents the torque constant. With $50.77 \mathrm{mNm} / \mathrm{A}$, it lies close to the quotient of the starting current and the stall torque from the datasheet of $52.75 \mathrm{mNm} / \mathrm{A}$.

We determined the static friction with the usual method and compared it to our former result. The current was increased until a movement occurred, determined by an encoder value unequal to zero. After repeating 100 runs, the measured threshold currents were averaged and resulted in a breakaway current of $120 \mathrm{~mA}$. The lower value comes from the encoder's high sensitivity in conjunction with the gear's backlash. Since the application of the higher threshold current onto the motor does not lead to unwanted continuous 


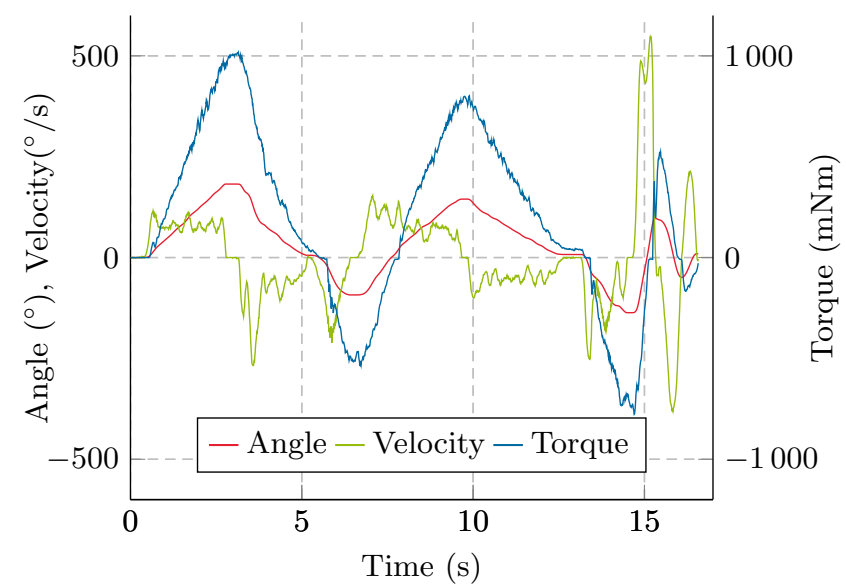

Figure 4: Representative short session in virtual spring mode.

movement, the higher value is valid. It can now be utilized to decrease the initial user induced torque and improve the accuracy of the torque estimation. We measured a rootmean-square error of $25.78 \mathrm{mNm}$ for the fitted dataset. Applied to the measurements from a second run, the error was only slightly higher with $30.08 \mathrm{mNm}$.

The torque control can be applied in different training modes. We implemented a virtual spring where the torque or the current set point, respectively, is linearly increased with the angle difference to a neutral position. A representative short session is shown in Fig. 4 with the angle, velocity, and torque plotted against the time.

\section{CONCLUSION}

A rehabilitation device for training wrist and finger functions has been proposed. The torque estimation based on current measurements and the angle sensing from the motor encoder readings permits virtual rehabilitation and studying rehabilitation paradigms. The performance evaluation revealed a relative root-mean-square error of $2.2 \%$ which is acceptable considering the reduction in costs achieved by omitting a force-torque sensor. Therefore, expensive sensors can be omitted without relinquishing torque control and measurement. However, closed-loop force control, e.g. necessary for admittance devices, is not possible. The Qt GUI can be run on a Rasperry $\mathrm{Pi}$ which offers full capabilities of a PC for less than $\$ 50$. The device can be accessed remotely and session data can be transmitted to therapists using web services. The cost-effective therapy device provides remote access capabilities which are important prerequisites for home rehabilitation. In future studies, we intend to validate the applicability, efficacy and reliability of the system with stroke patients.

\section{REFERENCES}

[1] J. Allington, S. J. Spencer, J. Klein, M. Buell, D. J. Reinkensmeyer, and J. Bobrow. Supinator extender (sue): a pneumatically actuated robot for forearm/wrist rehabilitation after stroke. In Engineering in Medicine and Biology Society, EMBC, 2011 Annual International Conference of the IEEE, pages 1579-1582. IEEE, 2011.

[2] S. Balasubramanian, J. Klein, and E. Burdet. Robot-assisted rehabilitation of hand function. Current opinion in neurology, 23(6):661, 2010.

[3] B. R. Brewer, R. Klatzky, and Y. Matsuoka. Visual feedback distortion in a robotic environment for hand rehabilitation. Brain research bulletin, 75(6):804-813, 2008.

[4] S. Hesse, G. Schulte-Tigges, M. Konrad, A. Bardeleben, and C. Werner. Robot-assisted arm trainer for the passive and active practice of bilateral forearm and wrist movements in hemiparetic subjects. Archives of physical medicine and rehabilitation, 84(6):915-920, 2003.

[5] P. L. Kolominsky-Rabas, P. U. Heuschmann, D. Marschall, M. Emmert, N. Baltzer, B. Neundörfer, O. Schöffski, K. J. Krobot, et al. Lifetime cost of ischemic stroke in germany: Results and national projections from a population-based stroke registry the erlangen stroke project. Stroke, 37(5):1179-1183, 2006.

[6] H. I. Krebs, B. T. Volpe, D. Williams, J. Celestino, S. K. Charles, D. Lynch, and N. Hogan. Robot-aided neurorehabilitation: a robot for wrist rehabilitation. Neural Systems and Rehabilitation Engineering, IEEE Transactions on, 15(3):327-335, 2007.

[7] G. Kwakkel, B. J. Kollen, and H. I. Krebs. Effects of robot-assisted therapy on upper limb recovery after stroke: a systematic review. Neurorehabilitation and neural repair, 22(2):111-121, 2008.

[8] P. Maciejasz, J. Eschweiler, K. Gerlach-Hahn, A. Jansen-Toy, S. Leonhardt, et al. A survey on robotic devices for upper limb rehabilitation. Journal of neuroengineering and rehabilitation, 11(1):3, 2014.

[9] J.-C. Metzger, O. Lambercy, D. Chapuis, and R. Gassert. Design and characterization of the rehapticknob, a robot for assessment and therapy of hand function. In Intelligent Robots and Systems (IROS), 2011 IEEE/RSJ International Conference on, pages 3074-3080. IEEE, 2011.

[10] H. Nakayama, H. S. Jgtgensen, H. O. Raaschou, and T. S. Olsen. Recovery of upper extremity function in stroke patients: the copenhagen stroke study. Age (SD), 74:11-2, 1994.

[11] J. Oblak, I. Cikajlo, and Z. Matjacic. Universal haptic drive: A robot for arm and wrist rehabilitation. Neural Systems and Rehabilitation Engineering, IEEE Transactions on, 18(3):293-302, 2010.

[12] D. J. Reinkensmeyer, J. L. Emken, and S. C. Cramer. Robotics, motor learning, and neurologic recovery. Annu. Rev. Biomed. Eng., 6:497-525, 2004.

[13] C. D. Takahashi, L. Der-Yeghiaian, V. Le, R. R. Motiwala, and S. C. Cramer. Robot-based hand motor therapy after stroke. Brain, 131(2):425-437, 2008.

[14] C. Warlow, J. Van Gijn, P. Sandercock, G. Hankey, M. Dennis, J. Bamford, J. Wardlaw, C. Sudlow, G. Rinkel, and P. Rothwell. Stroke: practical management. 2008.

[15] P. Weiss, M. Heldmann, T. Münte, A. Schweikard, and E. Maehle. A rehabilitation system for training based on visual feedback distortion. In Converging Clinical and Engineering Research on Neurorehabilitation, pages 297-302. Springer, 2013. 